\title{
On the asymptotic giant branch star origin of peculiar spinel grain OC2
}

\author{
M. Lugaro ${ }^{1}$, A. I. Karakas ${ }^{2}$, L. R. Nittler ${ }^{3}$, \\ C. M. O’D. Alexander ${ }^{3}$, P. Hoppe ${ }^{4}$, C. Iliadis ${ }^{5}$, and J. C. Lattanzio 6
}

1 Sterrenkundig Instituut, University of Utrecht, Postbus 80000, 3508 TA Utrecht, The Netherlands e-mail: M.Lugaro@phys.uu.nl

2 Origins Institute, Department of Physics \& Astronomy, McMaster University, Hamilton ON, Canada

3 Carnegie Institution of Washington, Department of Terrestrial Magnetism Washington DC 20015, USA

4 Max-Planck-Institute for Chemistry, PO Box 3060, 55020 Mainz, Germany

5 Department of Physics and Astronomy, 176 Phillips Hall, University of North Carolina, Chapel Hill, NC 27599-3255, USA

${ }^{6}$ Centre for Stellar and Planetary Astrophysics, School of Mathematical Sciences, Monash University, Victoria 3800, Australia

Received 7 June 2006 / Accepted 7 October 2006

\begin{abstract}
Context. Microscopic presolar grains extracted from primitive meteorites have extremely anomalous isotopic compositions revealing the stellar origin of these grains. Multiple elements in single presolar grains can be analysed with sensitive mass spectrometers, providing precise sets of isotopic compositions to be matched by theoretical models of stellar evolution and nucleosynthesis.

Aims. The composition of presolar spinel grain OC2 is different from that of all other presolar spinel grains. In particular, large excesses of the heavy $\mathrm{Mg}$ isotopes are present and thus an origin from an intermediate-mass (IM) asymptotic giant branch (AGB) star was previously proposed for this grain. We discuss the $\mathrm{O}, \mathrm{Mg}, \mathrm{Al}, \mathrm{Cr}$ and $\mathrm{Fe}$ isotopic compositions of presolar spinel grain $\mathrm{OC} 2$ and compare them to theoretical predictions.

Methods. We use detailed models of the evolution and nucleosynthesis of AGB stars of different masses and metallicities to compare to the composition of grain OC2. We analyse the uncertainties related to nuclear reaction rates and also discuss stellar model uncertainties.

Results. We show that the isotopic composition of $\mathrm{O}, \mathrm{Mg}$ and $\mathrm{Al}$ in $\mathrm{OC} 2$ could be the signature of an AGB star of IM and metallicity close to solar experiencing hot bottom burning, or of an AGB star of low mass (LM) and low metallicity $(\simeq 0.004)$ suffering very efficient cool bottom processing. Large measurement uncertainty in the Fe isotopic composition prevents us from discriminating which model better represents the parent star of OC2. However, the Cr isotopic composition of the grain favors an origin in an IM-AGB star of metallicity close to solar.

Conclusions. Our IM-AGB models produce a self-consistent solution to match the composition of OC2 within the uncertainties related to reaction rates. Within this solution we predict that the ${ }^{16} \mathrm{O}(p, \gamma){ }^{17} \mathrm{~F}$ and the ${ }^{17} \mathrm{O}(p, \alpha){ }^{14} \mathrm{~N}$ reaction rates should be close to their lower and upper limits, respectively. By finding more grains like $\mathrm{OC} 2$ and by precisely measuring their $\mathrm{Fe}$ and $\mathrm{Cr}$ isotopic compositions, it may be possible in the future to derive constraints on massive AGB models from the study of presolar grains.
\end{abstract}

Key words. nuclear reactions, nucleosynthesis, abundances - meteors, meteoroids - stars: AGB and post-AGB

\section{Introduction}

Presolar grains were born in circumstellar regions around ancient stars, ejected into the interstellar medium, preserved during the formation of the Solar System, and trapped inside primitive meteorites from which they are now extracted and analysed by various microanalytical techniques. Their isotopic compositions are extremely anomalous compared to those found in the bulk of materials formed in the Solar System (e.g., Earth, meteorites, etc.). They represent a detailed record of the compositions of their parent stars, and, as such, provide major constraints for models of stellar structure and nucleosynthesis and of the chemical evolution of the Galaxy (Zinner 1998; Clayton \& Nittler 2004; Lugaro 2005). Until recently, Mg-rich presolar grains have been thought to be rare, as the dominant identified carbide ( $\mathrm{SiC}$, graphite) and oxide (corundum: $\mathrm{Al}_{2} \mathrm{O}_{3}$ ) phases have low $\mathrm{Mg}$ contents. The situation changed with the advent of a new type of ion microprobe with improved spatial resolution and sensitivity, the Cameca NanoSIMS 50 (Hillion et al. 1994). Using the
NanoSIMS, Zinner et al. (2003) found that presolar spinel is, in fact, more abundant in meteorites than is presolar corundum, but has a finer grain size, with most grains having diameters less than $1 \mu \mathrm{m}$

A presentation and discussion of the properties and the compositions of the more than 300 presolar spinel grains reported so far can be found in Zinner et al. (2005) and Nguyen et al. (2003). Most presolar oxide as well as silicate grains show enhancements in ${ }^{17} \mathrm{O}$ and depletions in ${ }^{18} \mathrm{O}$ similarly to what is observed in red giant and low-mass (LM) AGB stars, pointing to such an origin for the majority of these grains (Nittler et al. 1997). These compositions are explained by the effect of the first dredge-up and extra-mixing processes. We focus in this paper on the composition of a single extraordinary presolar spinel grain, named OC2. OC2, of size $\approx 0.8 \mu \mathrm{m}$ (Fig. 1), was identified in a mixed acid residue of the Semarkona, Krymka and Bishunpur unequilibrated ordinary chondrites (Nittler \& Alexander 1999; Zinner et al. 2005). The isotopic composition of this grain (Table 1) is peculiar among presolar oxide grains. Its most remarkable 


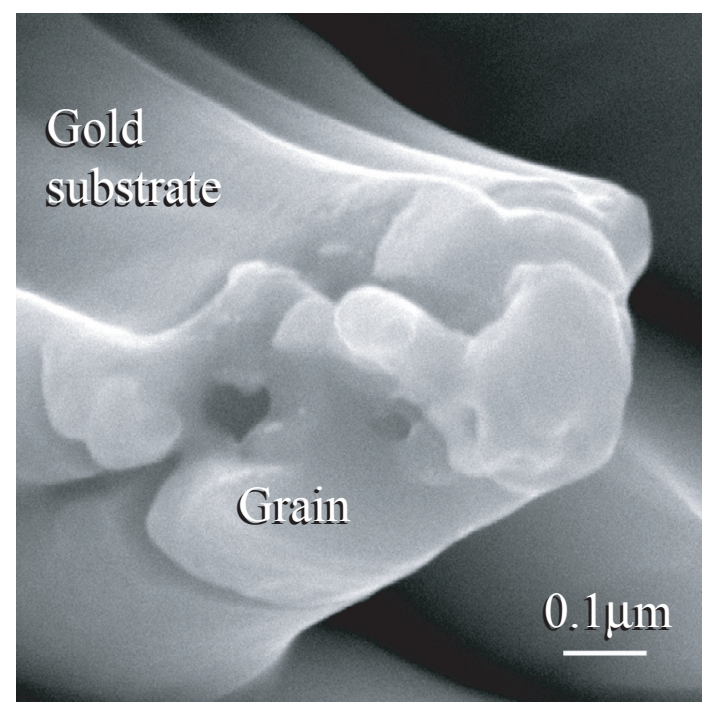

Fig. 1. Scanning electron microscope image of presolar spinel grain OC2. This $800 \mathrm{~nm}$ grain is sitting on a gold pedestal, following the ion probe isotopic analysis, because the gold substrate sputters faster than the grain does.

Table 1. The measured composition of presolar spinel grain OC2.

\begin{tabular}{lcc}
\hline \hline & OC2 & Solar ratio \\
\hline$\delta\left({ }^{25} \mathrm{Mg} /{ }^{24} \mathrm{Mg}\right)$ & $433.0 \pm 10.0$ & 0.1266 \\
$\delta\left({ }^{26} \mathrm{Mg} /{ }^{24} \mathrm{Mg}\right)$ & $1170.0 \pm 15.0$ & 0.1393 \\
$\mathrm{Al} / \mathrm{Mg}$ & $2.18 \pm 0.39$ & 0.079 \\
${ }^{17} \mathrm{O} /{ }^{16} \mathrm{O}$ & $1.25 \pm 0.07 \times 10^{-3}$ & $3.83 \times 10^{-4}$ \\
${ }^{18} \mathrm{O} /{ }^{16} \mathrm{O}$ & $6.94 \pm 1.34 \times 10^{-5 *}$ & $2.00 \times 10^{-3}$ \\
$\delta\left({ }^{57} \mathrm{Fe} /{ }^{56} \mathrm{Fe}\right)$ & $170 \pm 191$ & 0.0231 \\
$\delta\left({ }^{50} \mathrm{Cr} /{ }^{52} \mathrm{Cr}\right)$ & $26 \pm 71$ & 0.0519 \\
$\delta\left({ }^{53} \mathrm{Cr} /{ }^{52} \mathrm{Cr}\right)$ & $-56 \pm 45$ & 0.1135 \\
$\delta\left({ }^{54} \mathrm{Cr} /{ }^{52} \mathrm{Cr}\right)$ & $102 \pm 117$ & 0.0282 \\
\hline
\end{tabular}

All errors are given at $1 \sigma .{ }^{*}$ Quoted here is the experimental statistical uncertainty, which does not take into account the possible effect of pollution of terrestrial material discussed in Sect. 3.

feature are large excesses of the heavy $\mathrm{Mg}$ isotopes: ${ }^{25} \mathrm{Mg}$ and ${ }^{26} \mathrm{Mg}$ are enriched with respect to solar composition by $43 \%$ and $117 \%$, respectively (Fig. 2). The Mg isotopic composition is represented in Table 1 by the $\delta$ notation:

$$
\delta\left({ }^{i} \mathrm{Mg} /{ }^{24} \mathrm{Mg}\right)=\left(\frac{\left({ }^{i} \mathrm{Mg} /{ }^{24} \mathrm{Mg}\right)_{\text {measured }}}{\left({ }^{i} \mathrm{Mg} /{ }^{24} \mathrm{Mg}\right)_{\text {solar }}}-1\right) \times 1000,
$$

which we will use throughout this paper, where the abundant ${ }^{24} \mathrm{Mg}$, which represents about $79 \%$ of all magnesium in the Solar System, is used as the reference isotope. Note that the measured ${ }^{26} \mathrm{Mg} /{ }^{24} \mathrm{Mg}$ ratio includes both the abundance of ${ }^{26} \mathrm{Mg}$ itself and the contribution from ${ }^{26} \mathrm{Al}$ decay $\left(T_{1 / 2}=0.7\right.$ million $\left.\mathrm{yr}\right)$.

The $\mathrm{O}$ isotopic composition of grain OC2 (Fig. 3) is also quite extreme with respect to typical compositions of presolar oxide grains. While its ${ }^{17} \mathrm{O} /{ }^{16} \mathrm{O}$ ratio is enriched by a factor of 3.3 relative to solar, its ${ }^{18} \mathrm{O} /{ }^{16} \mathrm{O}$ ratio is at least 26 times smaller than that of the Solar System. Presolar oxides with large ${ }^{18} \mathrm{O}$ depletions have been classified as "Group 2" grains by Nittler et al. $(1994,1997)$, but these are in fact quite rare. Of some 600 known presolar corundum, hibonite $\left(\mathrm{CaAl}_{12} \mathrm{O}_{19}\right)$ and spinel grains, only 10 have ${ }^{18} \mathrm{O} /{ }^{16} \mathrm{O}$ ratios lower than $10^{-4}$, similar to that of OC2 (Fig. 3).

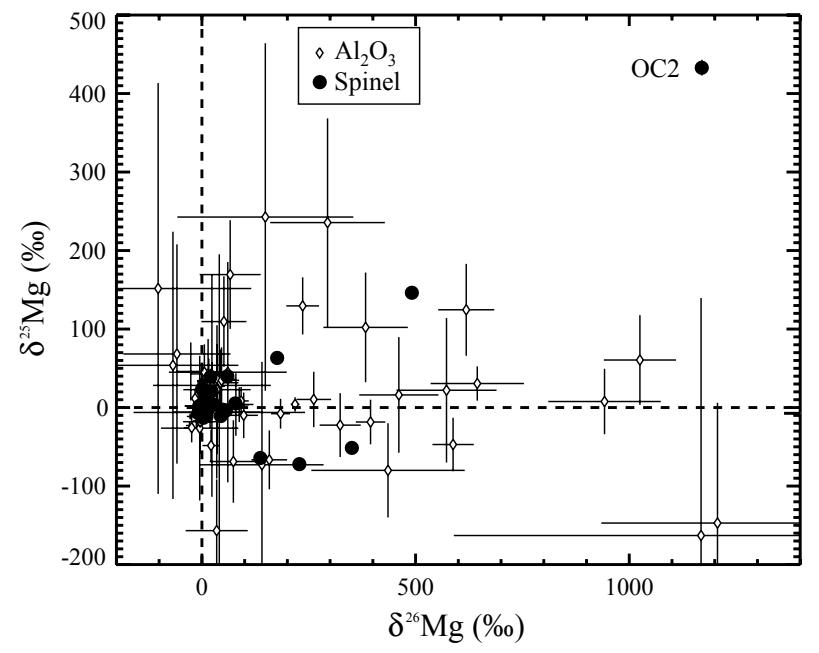

Fig. 2. Mg isotopes in presolar spinel and corundum grains (Nittler et al. 1997; Choi et al. 1999; Zinner et al. 2005). Dashed lines indicate solar isotopic ratios; errors are 1- $\sigma$. Spinel grain OC2 has larger excesses of ${ }^{25} \mathrm{Mg}$ and ${ }^{26} \mathrm{Mg}$ than seen in other presolar spinel grains. Measured $\delta\left({ }^{26} \mathrm{Mg} /{ }^{24} \mathrm{Mg}\right)$ values for corundum extend up to $1.7 \times 10^{6}$, due to high initial contents of ${ }^{26} \mathrm{Al}$ and high $\mathrm{Al} / \mathrm{Mg}$ ratios, but no corundum grain has a ${ }^{25} \mathrm{Mg}$ enrichment similar to that of OC2.

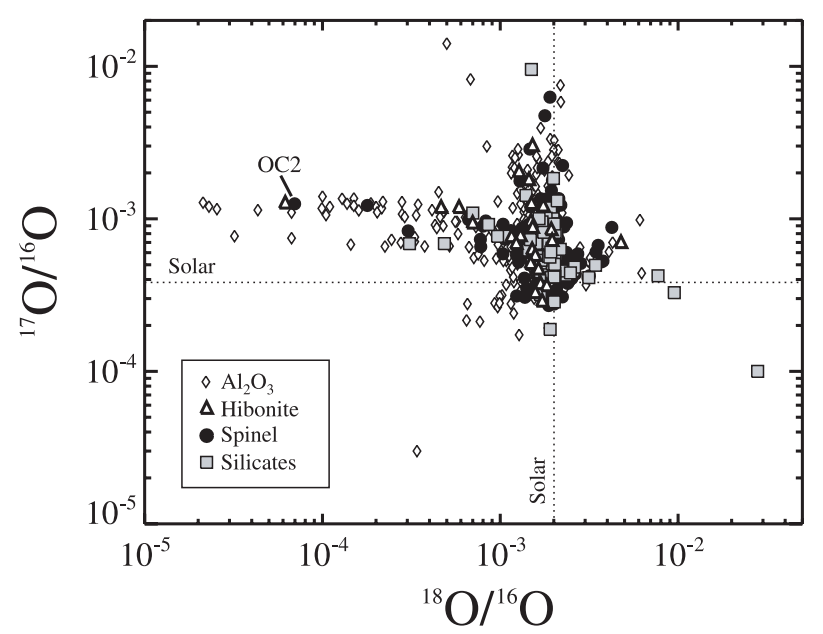

Fig. 3. O isotopic ratios measured in presolar corundum, hibonite, spinel, and silicate grains (Nittler 1997; Nittler et al. 1997; Choi et al. 1998; Messenger et al. 2003; Zinner et al. 2003; Mostefaoui \& Hoppe 2004; Nguyen \& Zinner 2004; Nittler et al. 2005). Grain OC2 is a member of the rare Group 2 class of highly ${ }^{18} \mathrm{O}$-depleted grains.

The $\mathrm{Cr}$ isotopic ratios were also measured in grain $\mathrm{OC} 2$, as well as the ${ }^{57} \mathrm{Fe} /{ }^{56} \mathrm{Fe}$ ratio. From Table 1 we see that these values are typically solar within the error bars.

The large ${ }^{25} \mathrm{Mg}$ and ${ }^{26} \mathrm{Mg}$ excesses observed in OC2 led Zinner et al. (2005) to propose for this grain an origin in an intermediate-mass (IM)-AGB star, with mass between 4-7 $M_{\odot}$ and underging hot bottom burning (Herwig 2005), instead of the usual low-mass origin assigned to the majority of the other presolar oxide grains. As for the late evolutionary phases of most stellar types, strong mass-loss and dust formation are observed around massive AGB stars. In fact, IM-AGB stars are part of the family of $\mathrm{OH} / \mathrm{IR}$ stars, i.e. O-rich AGB stars extremely bright in the infrared, and astronomical observations indicates that OH/IR stars are the second most important source of dust in the Galaxy (Alexander 1997; Whittet 1992). 
During the AGB phase, the $\mathrm{H}$ and He-burning shells are activated alternately in the deep layers of the star while the extended $\mathrm{H}$-rich envelope loses mass through strong stellar winds. During each He-burning episode, the sudden thermal runaway triggers the whole region between the $\mathrm{H}$ - and the He-shell (He intershell) to become convective. After a thermal pulse (TP) quenches, the base of the convective envelope can sink into the He intershell (third dredge-up, TDU), thus carrying to the surface nuclear processed material (see Herwig 2005, for a review on AGB stars).

The nucleosynthesis occurring in IM-AGB stars is different from that occurring in LM-AGB stars because the temperature during thermal pulses can exceed $\simeq 350$ million degrees and thus the heavy $\mathrm{Mg}$ isotopes can be produced by $\alpha$-capture reactions on ${ }^{22} \mathrm{Ne}$, which is abundantly present in the He intershell due to the conversion of ${ }^{14} \mathrm{~N}$ from $\mathrm{H}$-burning ashes into ${ }^{22} \mathrm{Ne}$ by double $\alpha$ captures during the early phases of a thermal pulse (Karakas et al. 2006b). The heavy Mg isotopes are then mixed to the envelope by the following TDU episode. In IM-AGB stars proton captures also occur at the base of the convective envelope (hot bottom burning, $\mathrm{HBB}$ ). The $\mathrm{MgAl}$ chain is activated resulting typically in the destruction of ${ }^{25} \mathrm{Mg}$ and the production of ${ }^{26} \mathrm{Al}$ and ${ }^{26} \mathrm{Mg}$. If the temperature exceeds $\simeq 80$ million degrees ${ }^{24} \mathrm{Mg}$ also suffers proton captures (see detailed discussion and models by Karakas \& Lattanzio 2003; Karakas et al. 2006b). $\mathrm{HBB}$ also greatly affects the $\mathrm{O}$ isotopic composition, resulting in the production of ${ }^{17} \mathrm{O}$ and the destruction of ${ }^{18} \mathrm{O}$, as observed in OC2. HBB can also prevent the surface of the star from becoming carbon-rich (Boothroyd et al. 1993), by converting the dredged-up ${ }^{12} \mathrm{C}$ to ${ }^{14} \mathrm{~N}$. Thus, IM-AGB stars might be expected to preferentially form oxide rather than carbonaceous phases like $\mathrm{SiC}$ and graphite, which require $\mathrm{C}>\mathrm{O}$. However, the formation of spinel cannot be completely ruled out for $\mathrm{C} / \mathrm{O}$ ratios slightly above unity (see discussion in Zinner et al. 2005).

Stellar sources of dust in the Galaxy other than AGB stars (see, e.g., Table 1 of Alexander 1997) seem unlikely to be the site of origin of grain OC2. The ejecta of supernova explosions are enriched in ${ }^{25} \mathrm{Mg},{ }^{26} \mathrm{Mg}$ and ${ }^{26} \mathrm{Al}$ when considering solar metallicity models. However, contrarily to the composition of OC2, ${ }^{16} \mathrm{O}$ and ${ }^{18} \mathrm{O}$ are produced, while ${ }^{17} \mathrm{O}$ is typically much depleted (Rauscher et al. 2002; Limongi \& Chieffi 2003). Wolf-Rayet stars, of which the WC type are also observed to generate dust (see e.g Williams et al. 1987), could also produce the O isotopic composition of grain OC2 together with excesses in ${ }^{26} \mathrm{Al}$ at a time just before the transition from $\mathrm{WN}$ to $\mathrm{WC}$ occurs (Arnould et al. 1997). However, no production of ${ }^{25} \mathrm{Mg}$ is expected, at least until core He burning starts and the star moves into the WC/WO phases, at which point also large depletions of ${ }^{17} \mathrm{O}$ and possible enhancements in ${ }^{18} \mathrm{O}$ are predicted. Nova nucleosynthesis is predicted to produce high enhancements in ${ }^{25} \mathrm{Mg},{ }^{26} \mathrm{Mg}$ and ${ }^{26} \mathrm{Al}$, however, these are typically accompanied by high enhancements also in both ${ }^{17} \mathrm{O}$ and ${ }^{18} \mathrm{O}$ (José et al. 2004). Only model CO1 of José et al. (2004), a CO nova of low mass 0.6 $M_{\odot}$, produces a deficit in ${ }^{18} \mathrm{O}$ comparable to that observed in grain OC2. However, the ${ }^{17} \mathrm{O} /{ }^{16} \mathrm{O}$ in this model is three times higher than in OC2, and the excesses in the heavy $\mathrm{Mg}$ isotopes are: $\delta\left({ }^{25} \mathrm{Mg} /{ }^{24} \mathrm{Mg}\right)=836$ and $\delta\left({ }^{26} \mathrm{Mg} /{ }^{24} \mathrm{Mg}\right.$ ) $=19062$ (without adding up the contribution of ${ }^{26} \mathrm{Al}$, with $\left.{ }^{26} \mathrm{Al} /{ }^{27} \mathrm{Al}=0.006\right)$ ! Even if mixing between this type of nova material and material of solar-like composition were invoked, it would be impossible to avoid producing ${ }^{26} \mathrm{Mg}$ excesses much higher than those observed in grain OC2 at the time when the ${ }^{25} \mathrm{Mg} /{ }^{24} \mathrm{Mg}$ ratio is matched.

The aim of this paper is to analyse the $\mathrm{O}, \mathrm{Mg}, \mathrm{Al}, \mathrm{Cr}$ and $\mathrm{Fe}$ isotopic compositions predicted by detailed models of AGB stars of different masses and metallicities and discuss them in the light of the precise measurements of the composition of grain OC2. In this way, we can test the idea that grain OC2 originated in an IM-AGB star. There are few direct constraints available to test theoretical models of these stars mostly owing to the fact that they are much rarer than their lower-mass counterparts. There are observations available for $\mathrm{Li}, \mathrm{C}, \mathrm{O},{ }^{12} \mathrm{C} /{ }^{13} \mathrm{C}$ and heavy elements abundances, in particular for AGB stars in the Magellanic Clouds (see e.g. Wood et al. 1983; Plez et al. 1993; Smith et al. 1995; van Loon et al. 1999), and recently also for AGB stars in our Galaxy (García-Hernández et al. 2006). The Li, C and O abundances and the ${ }^{12} \mathrm{C} /{ }^{13} \mathrm{C}$ ratios have been used as tests for the occurrence of HBB. Stellar models are able to explain the fact that the majority of AGB stars of high luminosity are $\mathrm{O}$ rich, as well as to reproduce the observed $l$ low ${ }^{12} \mathrm{C} /{ }^{13} \mathrm{C}$ ratios and high $\mathrm{Li}$ abundances (e.g. Boothroyd et al. 1993; Mazzitelli et al. 1999).

The paper is structured as follows: in Sect. 2 we describe the numerical method and the structure features of the stellar models presented in this paper. In Sect. 3 we compare the composition of grain OC2 to the compositions derived from our stellar models and discuss the model uncertainties. In Sect. 4 we outline our conclusions.

\section{Methods and models}

The evolution of stars of different masses and metallicities was computed from the zero-age main sequence to near the tip of the AGB using the Monash version of the Mt. Stromlo Stellar Structure code (see Frost \& Lattanzio 1996, and references therein for details). The models of IM-AGB stars that are used throughout this paper have masses of $5 M_{\odot}$ with $Z=0.02$ and 0.008 and $6.5 M_{\odot}$ with $Z=0.02$ and 0.012 . The $Z=0.02$ computations were assumed to have initial abundances taken from Anders \& Grevesse (1989), whereas the $Z=0.012$ models have initial abundances taken from Asplund et al. (2005) for elemental abundances and Lodders (2003) for initial isotopic ratios. Most presolar grains recovered from meteorites have come from LM-AGB stars so we also present results from one low-mass low- $Z$ AGB model with 2.5 Msun and $Z=0.004$. Models of AGB stars with $Z<0.004$ (or $Z=0.004$ in the case of IM-AGB stars) are not presented because presolar grains most likely originated in field stars from the solar neighborhood. While we do not have any reliable observational information on interstellar dust lifetimes, theoretical estimates give values $<1$ Gyr (Jones et al. 1997).

All models include mass loss on the AGB according to the prescription of Vassiliadis \& Wood (1993), show deep TDU and with the exception of the $2.5 M_{\odot}$ case, HBB, which prevents them from becoming $C$ rich. For the $5 M_{\odot} Z=0.02$ model we tested the effect of using the mass-loss rate from van Loon et al. (2005). We find that the two formulas produce the same mass-loss rate in the final phases of the evolution (the last 8 out of 24 pulses), which is consistent given that the formula of van Loon et al. (2005) is valid for the superwind phase of extreme mass loss in O-rich AGB stars. In Table 2 we present some features of the stellar models, where M5Z02 denotes the $5 M_{\odot}$ model with $Z=0.02$. We include the number of TPs, the core mass at the first TP, the total amount of matter dredgedup into the envelope, $M_{\text {dred }}^{\text {tot }}$, and the maximum dredge-up efficiency, $\lambda$ (for the definition, see Karakas et al. 2002), where all masses are given in solar units. We also present the maximum temperature at the base of the convective envelope, $T_{\mathrm{BCE}}^{\max }$, and in the He-intershell, $T_{\mathrm{He}}^{\max }$, both in units of $10^{6} \mathrm{~K}$; and the core and envelope mass at the final time-step. In all IM-AGB models, 
Table 2. Structural properties of the AGB models, see the text for details.

\begin{tabular}{lccccc}
\hline \hline Models: & M5Z02 & M6.5Z02 & M6.5Z012 & M5Z008 & M2.5Z004 \\
\hline No. TP & 24 & 40 & 51 & 59 & 34 \\
$M_{\mathrm{c}}(1)$ & 0.861 & 0.951 & 0.956 & 0.870 & 0.602 \\
$M_{\mathrm{dred}}^{\text {tot }}$ & $5.027(-2)$ & $4.696(-2)$ & $6.483(-2)$ & $1.745(-1)$ & $1.869(-1)$ \\
$\lambda_{\max }^{\max }$ & 0.961 & 0.910 & 0.940 & 0.952 & 0.820 \\
$T_{\mathrm{BCE}}^{\max }$ & 64 & 87 & 90 & 81 & - \\
$T_{\mathrm{He}}^{\max }$ & 352 & 372 & 370 & 366 & 308 \\
$M_{\text {env }}^{\mathrm{f}}$ & 1.499 & 1.507 & 1.389 & 1.387 & 0.685 \\
$M_{\text {core }}^{\mathrm{f}}$ & 0.874 & 0.963 & 0.967 & 0.886 & 0.673 \\
\hline
\end{tabular}

HBB was shut off as a consequence of mass loss. Once the envelope mass drops below about $1.5 M_{\odot}$ the temperature rapidly drops below that required to sustain proton capture nucleosynthesis (Karakas et al. 2006b). Dredge-up may continue, adding further ${ }^{12} \mathrm{C}$ to the envelope and, depending on the initial metallicity, increase the $\mathrm{C} / \mathrm{O}$ to $\geq 1$ (Frost et al. 1998; Karakas et al. 2006b). From Karakas et al. (2006b), however, this only occurs in IM-AGB stars with $Z \leq 0.004$.

We performed detailed nucleosynthesis calculations using a post-processing code which includes 74 species, 506 reactions and time-dependent diffusive mixing in all convective zones (Cannon 1993). Details on the post-processing code and network are outlined in Lugaro et al. (2004b) and Karakas et al. (2006b) and will not be repeated here. Details on the reaction rates can be found in Lugaro et al. (2004b), where it is outlined which of the proton, $\alpha$ and neutron capture reaction rates we have updated (from the REACLIB data tables of Thielemann et al. 1986, 1991 version), according to the latest experimental results. More recent updates include the ${ }^{22} \mathrm{Ne}+\alpha$ reaction rates (Karakas et al. 2006b) and the NACRE rates (Angulo et al. 1999, NACRE) for the proton captures rates of the $\mathrm{NeNa}$ and $\mathrm{MgAl}$ chains. The case of the ${ }^{17} \mathrm{O}(p, \alpha){ }^{14} \mathrm{~N}$ reaction, of particular importance for the present work, is discussed in Sect. 3.1.

For the $5 M_{\odot} Z=0.008$ and $2.5 M_{\odot} Z=0.004$ models, we assume an initial $\alpha$-enhanced mixture for the elements $\mathrm{O}, \mathrm{Ne}$, $\mathrm{Mg}, \mathrm{Si}$ and S typical of thin-disk stars (Reddy et al. 2003). The initial composition of the $\mathrm{O}$ and $\mathrm{Mg}$ isotopes are determined by the $\alpha$-enhancement of ${ }^{16} \mathrm{O}$ and ${ }^{24} \mathrm{Mg}$, so that $[\mathrm{O} / \mathrm{Fe}]=+0.4$ and $[\mathrm{Mg} / \mathrm{Fe}]=+0.27$ at $[\mathrm{Fe} / \mathrm{H}]=-1$, while the abundances of the neutron-rich isotopes are scaled with the initial metallicity. Thus, the ${ }^{17} \mathrm{O} /{ }^{16} \mathrm{O}$ and ${ }^{18} \mathrm{O} /{ }^{16} \mathrm{O}$ ratios are lower than their solar values (see Table 1), by factors 1.44 and 1.91 at $Z=0.008$ and 0.004 , respectively. With this choice we obtain, at any given metallicity, higher O ratios than suggested by Timmes et al. (1995) by following the Galactic chemical evolution, and where the $\mathrm{O}$ ratios are scaled with the metallicity. However, there are still large uncertainties in the evolution of the $\mathrm{O}$ isotopic ratios with time (Prantzos et al. 1996; Romano \& Matteucci 2003). Most importantly for the discussion here, the effect of the first and second dredge-up mixing events and $\mathrm{HBB}$ almost completely erases any record of the initial $\mathrm{O}$ ratios at the surface during the TP-AGB phase.

\section{Comparison of model predictions and the composition of $\mathrm{OC} 2$}

In Fig. 4 the $\mathrm{Mg}$ isotopic composition of grain OC2 is compared to that predicted by our calculations of IM-AGB models. The predictions shown in this plot are calculated by including the abundance of ${ }^{26} \mathrm{Al}$ multiplied by 25 , taking into account the fact that $\mathrm{Al}$ was incorporated in spinel grains during their

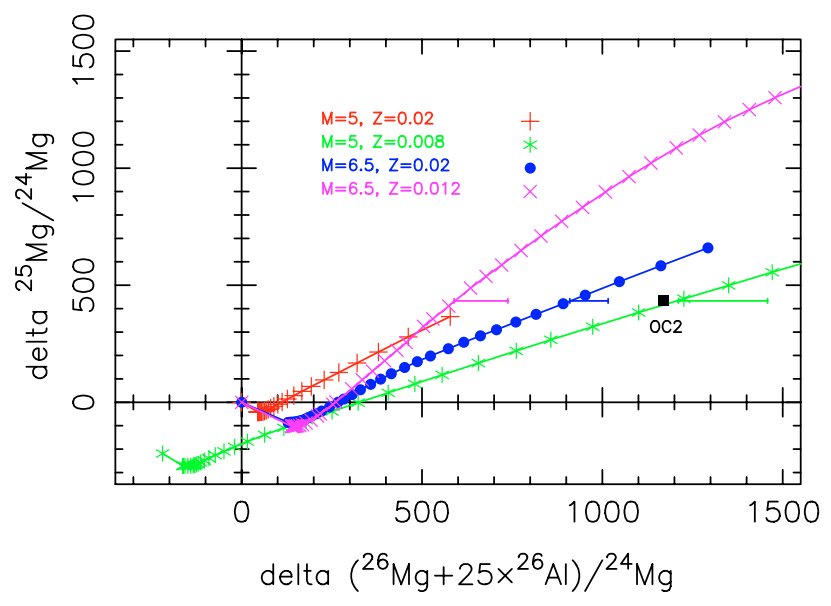

Fig. 4. The Mg isotopic compositions of grain OC2 is compared to our models of IM-AGB stars. The $2 \sigma$ uncertainties for OC2 are roughly within the symbol. Each symbol for model predictions represents the composition after a TDU episode. As indicated in the $\times$-label the $\delta\left({ }^{26} \mathrm{Mg} /{ }^{24} \mathrm{Mg}\right)$ measured in OC2 is compared to prediction lines calculated by including the abundance of ${ }^{26} \mathrm{Al}$ multiplied by a factor of 25 to take into account the fact that $\mathrm{Al}$ is preferentially included in spinel by such factor (see text). The uncertainty range in the predictions derived from the calculation of this factor are represented by the error bars connected to each prediction line at $\delta\left({ }^{25} \mathrm{Mg} /{ }^{24} \mathrm{Mg}\right)=433$. Solar composition is represented by ticked axis at $\delta=0$.

formation approximately 25 times more preferentially than $\mathrm{Mg}$, given that stoichiometric spinel by definition is $\mathrm{MgAl}_{2} \mathrm{O}_{4}$, i.e. it has $\mathrm{Al} / \mathrm{Mg}=2$, while this ratio is 0.079 in the Solar System (Zinner et al. 2005). We have explored the uncertainty related to this ${ }^{26} \mathrm{Al}$ multiplication factor by considering the $\mathrm{Al} / \mathrm{Mg}$ ratios predicted at the stellar surface in our models at the time when $\delta\left({ }^{25} \mathrm{Mg} /{ }^{24} \mathrm{Mg}\right)_{\mathrm{OC} 2}$ is matched, which are $5 \%$ to $20 \%$ lower than in the Solar System, and the error bars of the $\mathrm{Al} / \mathrm{Mg}$ ratio measured in OC2 (see Table 1). The resulting uncertainties are represented by the error bars connected to the prediction lines in Fig. 4.

First, let us consider the $5 M_{\odot} Z=0.02$ model, which does not reach the excess in ${ }^{25} \mathrm{Mg}$ shown by OC2. The ${ }^{25} \mathrm{Mg} /{ }^{24} \mathrm{Mg}$ ratio is determined by the combined effect of the He intershell temperatures and the total amount of material mixed to the surface by the TDU. An increase in either of those two quantities would lead to higher ${ }^{25} \mathrm{Mg} /{ }^{24} \mathrm{Mg}$ ratios, so the OC2 data indicate that an IM-AGB parent star of this grain must have experienced either temperatures higher than 352 million degrees, or a TDU mass higher than $0.05 M_{\odot}$, or both. Second, it appears that a narrow range of $\mathrm{HBB}$ temperatures is required in order to produce enough ${ }^{26} \mathrm{Al}$ to match the excess at mass 26 shown by $\mathrm{OC} 2$ at the given $\delta\left({ }^{25} \mathrm{Mg} /{ }^{24} \mathrm{Mg}\right)$ value, avoiding an increase of the $\delta\left({ }^{25} \mathrm{Mg} /{ }^{24} \mathrm{Mg}\right)$ value itself. This occurs in the models of $5 M_{\odot}$ and $Z=0.008$ metallicity and $6.5 M_{\odot}$ and $Z=0.02$ metallicity (in this latter case within reaction rate uncertainties, see Sect. 3.1), where the maximum temperature at the base of the convective envelope reaches 81 and 87 million degrees, respectively. For the $5 M_{\odot} Z=0.02$ case the temperature is too low, $\leq 64$ million degrees, to produce ${ }^{26} \mathrm{Al}$, while in the $6.5 M_{\odot}$ $Z=0.012$ case the temperature is too high, $\leq 90$ million degrees, so that also ${ }^{25} \mathrm{Mg}$ is produced by HBB. In principle, it is possible that for temperatures somewhat higher than 90 million degrees one could achieve again the composition of OC2 via HBB, as more ${ }^{25} \mathrm{Mg}$ is converted into ${ }^{26} \mathrm{Al}$. However, these temperatures are not achieved in our models, and, moreover, they would pose 


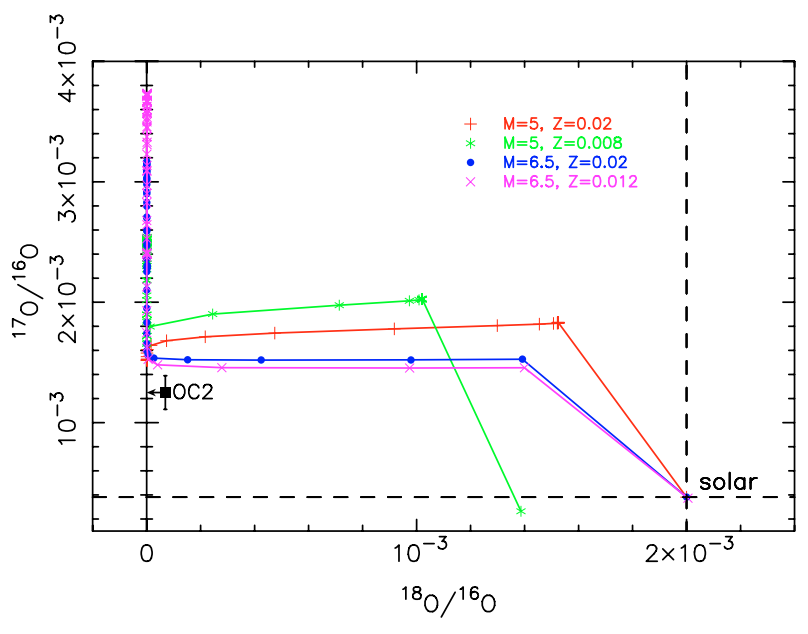

Fig. 5. The $\mathrm{O}$ isotopic compositions of grain $\mathrm{OC} 2$ is compared to predictions from our IM-AGB stars. The $2 \sigma$ uncertainties for the ${ }^{17} \mathrm{O} /{ }^{16} \mathrm{O}$ ratio of OC2 are indicated by the error bar, while for the ${ }^{18} \mathrm{O} /{ }^{16} \mathrm{O}$ ratio they are roughly within the symbol. The arrow indicates the effect of possible contamination of terrestrial material, as discussed in the text. Solar ratios are indicated by long-dashed lines.

a problem to match the ${ }^{17} \mathrm{O} /{ }^{16} \mathrm{O}$ ratio of the grain (see discussion below and in Sect. 3.1).

Note that the $\delta\left({ }^{25} \mathrm{Mg} /{ }^{24} \mathrm{Mg}\right)$ and $\delta\left({ }^{26} \mathrm{Mg} /{ }^{24} \mathrm{Mg}\right)$ values for the $5 M_{\odot} Z=0.008$ model reach values higher than 2000 and 5000, respectively, outside the range shown in Fig. 4. (For the $6.5 M_{\odot}$ $Z=0.012$ model, also outside the range of the figure, $\delta$-values reach $\simeq 2000$ and $\simeq 2500$, respectively). This could represent a difficulty for matching the composition of grain OC2 using the $5 M_{\odot} Z=0.008$ model. It is much more likely for a grain to be produced with a composition that corresponds to abundances in the envelope at the end of the AGB. This is because radio and infrared observations confirm that mass-loss rates of AGB stars increase with time and that these stars expel a large part of their envelope towards the end of their evolution via a strong superwind, with $\dot{M} \sim$ few $10^{-4} M_{\odot} /$ yr (e.g. Vassiliadis \& Wood 1993; van Loon et al. 2005), leading to the formation of a planetary nebula. In the case of our $5 M_{\odot} Z=0.008$ model, about $40 \%$ of the mass is lost in the last few TPs when the Mg isotopic ratios are well above that observed in OC2. Instead, the mass lost in between the TDU episodes that cover the composition of OC2 is only $\simeq 0.005 M_{\odot}$, hence, the probability of a grain forming with such composition is much smaller, $\simeq 0.1 \%$, even if this occurrence cannot be ruled out. From this point of view, the $6.5 M_{\odot}$ $Z=0.02$ model could be favored for the parent star of OC2, since it reaches the observed $\delta\left({ }^{25} \mathrm{Mg} /{ }^{24} \mathrm{Mg}\right)$ towards the end of its evolution and the $\delta\left({ }^{26} \mathrm{Mg} /{ }^{24} \mathrm{Mg}\right)$ value is within reaction rate uncertainties (see Sect. 3.1). However, it should be also kept in mind that the mass-loss prescription is one of the largest uncertainties in AGB models, as discussed in Sect. 3.2.

In Fig. 5, the O isotopic composition of grain OC2 is compared to that predicted by our IM-AGB models, and in Fig. 6 the ${ }^{17} \mathrm{O} /{ }^{16} \mathrm{O}$ ratio is plotted as function of the $\delta\left({ }^{25} \mathrm{Mg} /{ }^{24} \mathrm{Mg}\right)$ value. These figures show that the ${ }^{17} \mathrm{O} /{ }^{16} \mathrm{O}$ ratio of grain $\mathrm{OC} 2$ is not matched by any of the models, as they always produce too high a ratio. As discussed by Nollett et al. (2003), the ${ }^{17} \mathrm{O} /{ }^{16} \mathrm{O}$ equilibrium ratio during proton captures is mainly determined by the ratio of the rates of the nuclear reactions that produce and destroy ${ }^{17} \mathrm{O}$, i.e. the ${ }^{16} \mathrm{O}(p, \gamma){ }^{17} \mathrm{~F}$ and the $\left.{ }^{17} \mathrm{O}(p, \alpha)\right)^{14} \mathrm{~N}$ reactions, respectively. This ratio reaches a minimum of $\simeq 0.0011$ around 50 million degrees and then increases again for higher temperatures

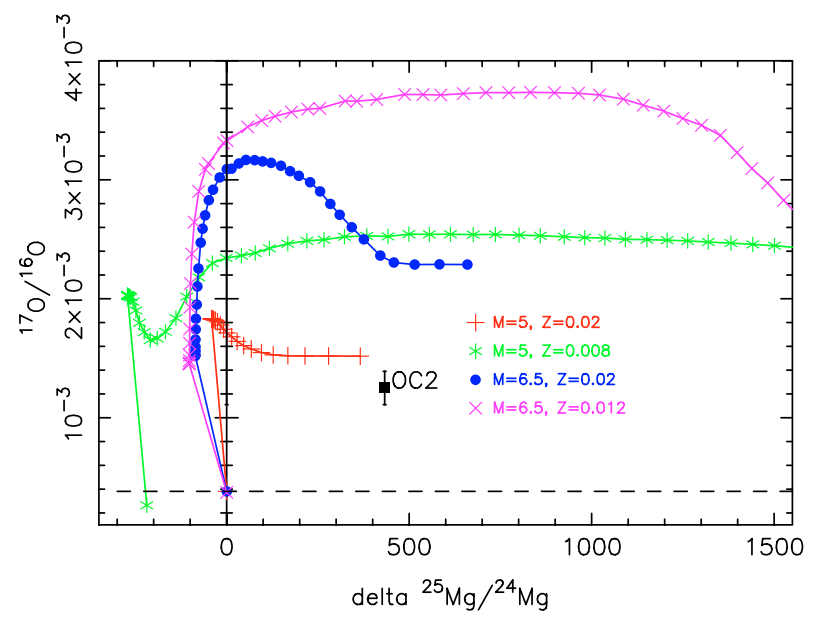

Fig. 6. The ${ }^{17} \mathrm{O} /{ }^{16} \mathrm{O}$ ratio is plotted as function of the $\delta\left({ }^{25} \mathrm{Mg} /{ }^{24} \mathrm{Mg}\right)$ for our IM-AGB models.

reaching 0.008 at 100 million degrees (see Fig. 8 of Nollett et al. 2003). During $\mathrm{HBB}$, the ${ }^{17} \mathrm{O} /{ }^{16} \mathrm{O}$ equilibrium ratio is reached at the base of the convective envelope and the envelope material is efficiently replaced by material with the ${ }^{17} \mathrm{O} /{ }^{16} \mathrm{O}$ equilibrium ratio after $\sim 5$ TPs. The $5 M_{\odot} Z=0.02$ model comes closest to producing the needed ratio because of its lower temperature, however, this model is the furthest from matching the measured $\delta\left({ }^{26} \mathrm{Mg} /{ }^{24} \mathrm{Mg}\right)$ value. The high temperatures at which HBB operates in the $5 M_{\odot} Z=0.008$ and in the $6.5 M_{\odot} Z=0.02$ models lead to an even worse match with the $\mathrm{O}$ isotopic composition of grain OC2. The $6.5 M_{\odot} Z=0.012$ model follows the expected trend as the ${ }^{17} \mathrm{O} /{ }^{16} \mathrm{O}$ is the highest in this case.

As for the ${ }^{18} \mathrm{O} /{ }^{16} \mathrm{O}$ ratio, because of $\mathrm{HBB}$, all the models reach ratios of the order of $10^{-6}-10^{-7}$, much lower than that shown by grain OC2, after only about 5 to 15 thermal pulses, much earlier than when the required $\mathrm{Mg}$ isotopic composition is reached. So, it is not possible to match the observed value by any of our models, but we note that there is always surface contamination on sample mounts and residual oxygen in the ion microprobe vacuum system. The very low measured ${ }^{18} \mathrm{O} /{ }^{16} \mathrm{O}$ ratio for grain OC2 was based on 35 actual counted ${ }^{18} \mathrm{O}$ atoms. If the grain actually had an ${ }^{18} \mathrm{O} /{ }^{16} \mathrm{O}$ ratio of zero, this low measured ${ }^{18} \mathrm{O}$ signal would correspond to a $2 \%$ level of terrestrial contamination, which is perfectly reasonable. Thus, we consider it likely that the true ${ }^{18} \mathrm{O} /{ }^{16} \mathrm{O}$ ratio of OC2 was indeed lower, and do not consider the mis-match with HBB models to be a major problem.

\subsection{The effect of varying the reaction rates}

As discussed above, the ${ }^{17} \mathrm{O} /{ }^{16} \mathrm{O}$ ratio only depends on the ratio of the ${ }^{16} \mathrm{O}(p, \gamma){ }^{17} \mathrm{~F}$ and the ${ }^{17} \mathrm{O}(p, \alpha){ }^{14} \mathrm{~N}$ reaction rates, so it is important to carefully evaluate the uncertainties related to these reaction rates in the temperature range of $\mathrm{HBB}, \simeq 60$ to 100 million degrees. The ${ }^{17} \mathrm{O}(p, \alpha){ }^{14} \mathrm{~N}$ reaction rate in this temperature range is almost completely determined by a resonance at $70 \mathrm{keV}$. In the models presented above we have used the rate from Blackmon et al. (1995) and Landré et al. (1990), which is the same as in the NACRE compilation. We have also calculated a new rate for this reaction on the basis of the latest available experimental information (Chafa et al. 2005; Fox et al. 2005). We have found a rate close to NACRE (within a few percent, for the temperature of interest in this study) with an uncertainty range of $\simeq+25 \%$ and $-30 \%$, compared to the NACRE ranges 


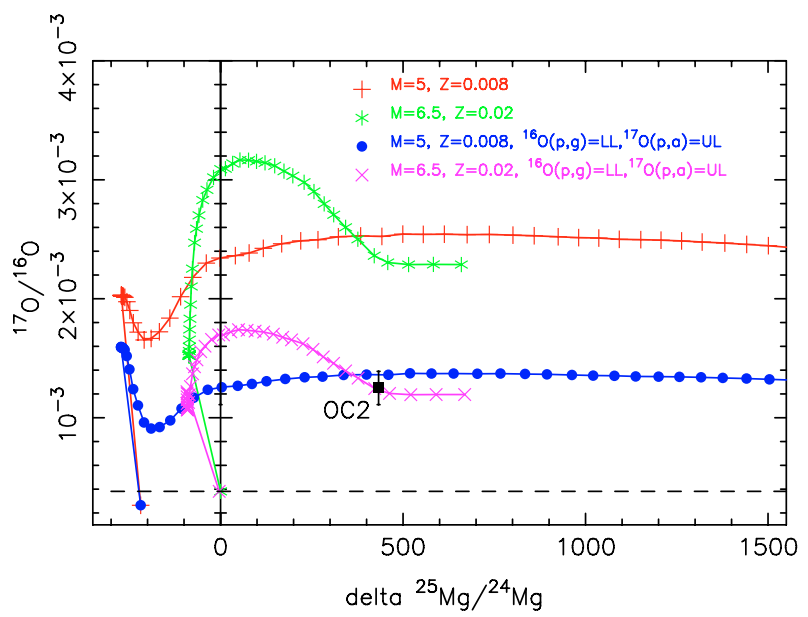

Fig. 7. Selected IM-AGB model predictions from Fig. 6 are shown, with extra model predictions calculated using the lower limit (LL) and the upper limit (UL) for the ${ }^{16} \mathrm{O}(p, \gamma){ }^{17} \mathrm{~F}$ and the ${ }^{17} \mathrm{O}(p, \alpha){ }^{14} \mathrm{~N}$ reaction rates, respectively.

of $\simeq+33 \%$ and $-22 \%$. The ${ }^{16} \mathrm{O}(p, \gamma){ }^{17} \mathrm{~F}$ rate is mostly determined by direct capture and the astrophysical $S$-factor at zero energy is extrapolated from the available data using a given potential model (Angulo et al. 1999). At low energy the experimental data show large error bars and the NACRE compilation adopts a $30 \%$ uncertainty for the derived $S$-factor. This results in lower and upper limits for the rate a factor 1.43 lower and 1.30 higher, respectively, than the recommended value at the temperature of interest. As shown in Fig. 7, the use of the upper limit for the ${ }^{16} \mathrm{O}(p, \gamma){ }^{17} \mathrm{~F}$ rate together with the lower limit for the ${ }^{17} \mathrm{O}(p, \alpha){ }^{14} \mathrm{O}$ rate is predicted to provide a good match to OC2, using the same models $\left(5 M_{\odot}, Z=0.008\right.$ and $6.5 M_{\odot}$, $Z=0.02$ ) that match its $\mathrm{Mg}$ isotopic composition.

The $\mathrm{Mg}$ isotopic composition is affected by the uncertainties in the ${ }^{22} \mathrm{Ne}+\alpha$ reaction rates as well as proton-capture reaction rates. The uncertainties of the ${ }^{22} \mathrm{Ne}+\alpha$ reaction rates have been recently re-evaluated by Karakas et al. (2006b) and are relatively small (of the order of $\simeq 60 \%$ ), while the uncertainties on the ${ }^{25} \mathrm{Mg}(p, \gamma)^{26} \mathrm{Al}$ and ${ }^{26} \mathrm{Al}(p, \gamma){ }^{27} \mathrm{Si}$ reaction rates are large (a factor of 3 and a factor of 1200, respectively) and affect, in particular, the production of ${ }^{26} \mathrm{Al}$. If we apply the uncertainties found by Izzard et al. (2006) to the ${ }^{26} \mathrm{Al}$ surface abundances of our models we find that the lower limit for the ${ }^{26} \mathrm{Al}$ yield, is incompatible with the composition of OC2, while the upper limit for the ${ }^{26} \mathrm{Al}$ yield would allow up to $\simeq 50 \%$ more ${ }^{26} \mathrm{Al}$ production. Combining this effect with the uncertainties of the ${ }^{22} \mathrm{Ne}+\alpha$ reaction rates (i.e. using the upper limit for the ${ }^{22} \mathrm{Ne}(\alpha, \gamma)^{26} \mathrm{Mg}$ and the lower limit for the ${ }^{22} \mathrm{Ne}(\alpha, n)^{25} \mathrm{Mg}$ reaction rates, respectively), we find that our $6.5 M_{\odot}, Z=0.02$ model can also provides a match to the $\mathrm{Mg}$ isotopic composition of OC2.

As a final remark we note that our stellar structure calculations have been performed using a ${ }^{14} \mathrm{~N}(p, \gamma){ }^{15} \mathrm{O}$ reaction rate very similar to NACRE. However, direct experimental data are now available for this rate down to the typical H-burning temperatures of AGB stars (Runkle et al. 2005; LUNA collaboration 2006). They give a rate $40 \%$ lower than NACRE. This has been shown to have an impact on the structure of AGB stars, in particular Weiss et al. (2005) have shown that for a $5 M_{\odot}$ star of solar metallicity the peak luminosity in thermal pulses is higher, and the interpulse duration longer, while Herwig \& Austin (2004) and Herwig (2006) have shown that for a $2 M_{\odot}$ star of $Z=0.01$ the choice of the new ${ }^{14} \mathrm{~N}(p, \gamma){ }^{15} \mathrm{O}$ reaction rate results in a more efficient third dredge-up. Further analysis is needed to test the effect of this updated reaction rate on nucleosynthesis in IM-AGB stars.

\subsection{The effect of model uncertainties}

One of the main uncertainties in AGB models is the choice of mass loss: a definitive agreement on the description of this phenomena is still missing. Ventura \& D'Antona (2005a) calculated models with different choices of mass loss and showed that a stronger mass loss leads to less efficient HBB nucleosynthesis. In this case it may be more difficult to produce enough ${ }^{26} \mathrm{Al}$ to match the $\delta\left({ }^{26} \mathrm{Mg} /{ }^{24} \mathrm{Mg}\right)$ of $\mathrm{OC} 2$.

Another uncertainty in AGB star models relates to the possible mixing of protons from the $\mathrm{H}$-rich envelope into a tiny region at the top of He intershell (partial mixing zone, PMZ) at the end of each TDU episode, leading to the formation of a ${ }^{13} \mathrm{C}$ pocket. This mixing is needed in order to reproduce the enhancements observed in AGB stars of heavy elements produced by slow neutron captures (the $s$ process), with neutrons released by the ${ }^{13} \mathrm{C}(\alpha, n){ }^{16} \mathrm{O}$ reaction (Gallino et al. 1998). In the ${ }^{13} \mathrm{C}$ pocket, the $\mathrm{Mg}$ isotopic composition can be altered by neutron captures. However, for IM-AGB models, we previously demonstrated (Karakas et al. 2006b) that the presence of a ${ }^{13} \mathrm{C}$ pocket does not change the $\mathrm{Mg}$ isotopic ratios at the stellar surface because a reasonable value for the mass of the pocket is too small, $\simeq 10^{-4} M_{\odot}$, to produce any effect on the overall $\mathrm{Mg}$ nucleosynthesis.

Other important uncertainties in the stellar modeling regard how convection and convective borders are treated. Ventura \& D'Antona (2005b) use a "Full Spectrum of Turbulence" (FST) prescription for convective regions, while we use Mixing Length Theory (MLT) with $\alpha=1.7$. The FST approach leads to a smaller efficiency of the TDU and higher HBB temperatures.

We cannot yet make a direct comparison with these models as detailed nucleosynthesis is only available at the moment for stars of $Z=0.001$ with FST. As discussed by Herwig (2005) gravity waves or convective extra mixing at the base of the envelope and at the boundaries of the thermal pulse can affect the amount of TDU mass and also the temperature in the intershell. Herwig $(2004 a, b)$ employs a special scheme to allow for some time-dependent hydrodynamical overshoot at convective boundaries. He typically finds very deep TDU and also that the convective thermal pulse penetrates inside the $\mathrm{C}-\mathrm{O}$ degenerate core. Models are available for $Z=0.0001$, well below the metallicities we are considering here. Also in this case it remains to be analysed in detail if these models could provide a match to the composition of grain OC2.

Finally, we conclude our discussion of model uncertainties by noting that rotation and magnetic fields are not included in our models. These typically affect the occurrence of extramixing phenomena and have been studied for stars of masses lower (see e.g. Denissenkov \& VandenBerg 2003; Talon \& Charbonnel 2005), or higher (see e.g. Heger et al. 2000; Maeder \& Meynet 2005; Yoon \& Langer 2005) than the IM star we are considering here. To our knowledge, however, there are no published models of massive AGB stars with rotation. Rotation and magnetic fields are not 1D phenomena and simplifications and parameterizations are required to input these physics into 1D stellar structure codes. These simplifications will result in extra parameters and uncertainties above those already included in the simulations. For these reasons it is also valuable to compute models without rotation, even though the effects of these 


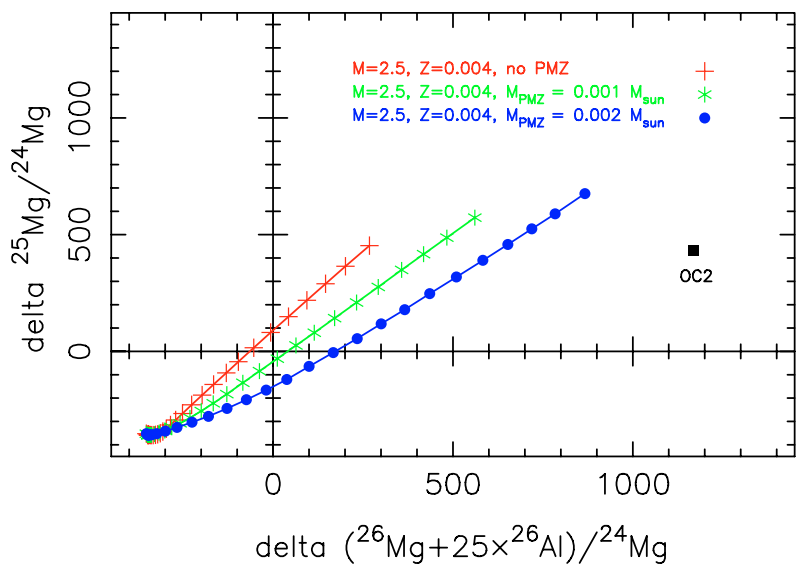

Fig. 8. The $\mathrm{Mg}$ predictions are shown for a $2.5 M_{\odot} Z=0.004$ model with different choices of the extension in mass of the partial mixing zone (PMZ).

phenomena on the evolution of massive AGB stars should be studied in the future.

\subsection{The case for a low-mass low-metallicity AGB star}

A LM-AGB star of mass $\simeq 2.5$ to $3.5 M_{\odot}$ and low metallicity, $Z \simeq 0.004$ to $\simeq 0.008$ can also produce the ${ }^{25} \mathrm{Mg}$ excess shown by OC2 because the He intershell temperature and the TDU efficiency are high enough in these stars. As an example, in Fig. 8 we present different models for a $2.5 M_{\odot}$ star of $Z=0.004$. As in the other figures, the predictions presented in this plot are calculated by including the abundance of ${ }^{26} \mathrm{Al}$ multiplied by the chosen factor of 25 , however in these models the contribution at mass 26 comes mostly from ${ }^{26} \mathrm{Mg}$. In two runs of the postprocessing of this model we artificially included the mixing of protons into the He intershell at the end of each TDU episode and we tested two different values for the extent in mass of the PMZ: 0.001 and $0.002 M_{\odot}$. More ${ }^{26} \mathrm{Mg}$ is produced when the pocket is included because of neutron captures. Since this star is just not hot enough for HBB to occur we have to assume that some extra-mixing process at the base of the convective envelope, such as the cool bottom processing (CBP) studied by Nollett et al. (2003), is at work during the AGB phase, so that enough ${ }^{26} \mathrm{Al}$ is produced and the observed $\delta\left({ }^{26} \mathrm{Mg} /{ }^{24} \mathrm{Mg}\right)$ is matched. In order to reproduce the $\delta\left({ }^{26} \mathrm{Mg} /{ }^{24} \mathrm{Mg}\right)$ of $\mathrm{OC} 2 \mathrm{a}^{26} \mathrm{Al} /{ }^{27} \mathrm{Al}$ ratio in the range 0.06 to 0.036 is required, which can be produced with $\mathrm{CBP}$ temperatures $\simeq 54$ million $\mathrm{K}$ (Nollett et al. 2003) The low ${ }^{18} \mathrm{O} /{ }^{16} \mathrm{O}$ ratio in $\mathrm{OC} 2$ is consistent with efficient $\mathrm{CBP}$ and the ${ }^{17} \mathrm{O} /{ }^{16} \mathrm{O}$ ratio would be 0.00115 at the $\mathrm{CBP}$ temperature derived from the ${ }^{26} \mathrm{Al} /{ }^{27} \mathrm{Al}$ ratio. Detailed calculations for $\mathrm{CBP}$ at low metallicities are required to test our present analysis before any strong conclusion can be drawn. We plan to include this process in our nucleosynthesis code in the future.

\subsection{The Fe and Cr isotopic composition}

In AGB stars the Fe isotopic composition is modified because the heavy $\mathrm{Fe}$ isotopes are produced by neutron captures occurring during the convective thermal pulses when the ${ }^{22} \mathrm{Ne}(\alpha, n){ }^{25} \mathrm{Mg}$ neutron source is activated. This production is rather insensitive to the neutron captures occurring in the ${ }^{13} \mathrm{C}$ pocket (Lugaro et al. 2004a). The $\delta\left({ }^{57} \mathrm{Fe} /{ }^{56} \mathrm{Fe}\right)$ value, at the time when $\delta\left({ }^{25} \mathrm{Mg} /{ }^{24} \mathrm{Mg}\right) \simeq 433$, is $\simeq 80$ in our IM-AGB models and $\simeq 370$ in the $2.5 M_{\odot} Z=0.004$ model. There are a couple of reasons for this difference: first, the integrated neutron flux increases with decreasing the metallicity as less material means that more free neutrons are available. Second, the dilution factor of the intershell material carried to the envelope by the TDU is about a factor of two higher in IM- than in LM-AGB stars. Unfortunately, the large uncertainty of the measured $\delta\left({ }^{57} \mathrm{Fe} /{ }^{56} \mathrm{Fe}\right)$ prevents us from determining which models represent the best match.

We cannot directly compare the $\mathrm{Cr}$ isotopic composition as we do not have the $\mathrm{Cr}$ isotopes in the network used to compute the present models. We can draw some general features on $\mathrm{Cr}$ isotopes in AGB stars considering Fig. 2 of Lugaro et al. (2004a), where results are presented for LM-AGB models of solar metallicity. The $\delta\left({ }^{50} \mathrm{Cr} /{ }^{52} \mathrm{Cr}\right)$ and $\delta\left({ }^{53} \mathrm{Cr} /{ }^{52} \mathrm{Cr}\right)$ are barely modified by neutron fluxes in AGB stars while the $\delta\left({ }^{54} \mathrm{Cr} /{ }^{52} \mathrm{Cr}\right)$ values are slightly higher than the $\delta\left({ }^{57} \mathrm{Fe} /{ }^{56} \mathrm{Fe}\right)$ values for a given stellar model. Since we are in the process of extending our network (Karakas et al. 2006a), using preliminary models we have checked that these results also apply to our IM-AGB models. In fact, for the $5 M_{\odot} Z=0.02$ model we obtain no changes in $\delta\left({ }^{50} \mathrm{Cr} /{ }^{52} \mathrm{Cr}\right)$ and $\delta\left({ }^{53} \mathrm{Cr} /{ }^{52} \mathrm{Cr}\right)$, while $\delta\left({ }^{54} \mathrm{Cr} /{ }^{52} \mathrm{Cr}\right)=170$, compared to $\delta\left({ }^{57} \mathrm{Fe} /{ }^{56} \mathrm{Fe}\right)=71$. For the $6.5 M_{\odot} Z=0.012$ model we obtain $\delta\left({ }^{54} \mathrm{Cr} /{ }^{52} \mathrm{Cr}\right)=40$, at pulse number 25 . Our IM-AGB models would match the $\delta\left({ }^{54} \mathrm{Cr} /{ }^{52} \mathrm{Cr}\right)$ value measured in $\mathrm{OC} 2$, while the $2.5 M_{\odot} Z=0.004$ model would produce $\delta\left({ }^{54} \mathrm{Cr} /{ }^{52} \mathrm{Cr}\right)$ values close to the $2 \sigma$ upper limit of OC2.

Finally, we note that in low metallicity stars the initial $\delta\left({ }^{i} \mathrm{Cr} /{ }^{52} \mathrm{Cr}\right)$ could be largely negative because of the effect of the Galactic Chemical Evolution of the $\mathrm{Cr}$ isotopes (see discussion in Zinner et al. 2005). This represents a hint against a LM-AGB star of low metallicity as the parent star for OC2, even though detailed models for the evolution of the $\mathrm{Cr}$ isotopes in the Galaxy should be performed to confirm this analysis.

\section{Conclusions}

We have shown that the peculiar isotopic composition of $\mathrm{O}, \mathrm{Mg}$ and $\mathrm{Al}$ in presolar spinel grain $\mathrm{OC} 2$ could be the signature of an AGB star of intermediate mass and metallicity close to solar (roughly higher than 0.008) suffering TDU and HBB, or of an AGB star of low mass and low metallicity (roughly lower than 0.008) suffering TDU and very efficient CBP. While HBB occurs in our IM-AGB models, we do not simulate any extramixing in the LM-AGB model. Thus, the LM-AGB origin for grain OC2 has still to be tested against detailed models including CBP in low metallicity AGB stars. The large uncertainty in the Fe isotopic composition of OC2 does not allow us to determine which model better represents the parent star of OC2, but the $\mathrm{Cr}$ isotopic composition favors an origin in an IM-AGB star of metallicity close to solar. In this case, the model conditions to reproduce the composition of OC2 are well defined: a TDU mass $>0.05 M_{\odot}$ and/or a maximum He-intershell temperature $>360$ million degrees, and a temperature at the base of the convective envelope in the range $\simeq 80$ to 85 million degrees. These conditions are satisfied by our $5 M_{\odot}, Z=0.008$ and $6.5 M_{\odot}, Z=0.02$ models. Within this solution, we predict that the ${ }^{16} \mathrm{O}(p, \gamma){ }^{17} \mathrm{~F}$ and the ${ }^{17} \mathrm{O}(p, \alpha){ }^{14} \mathrm{~N}$ reaction rates should be close to their lower and upper limits, respectively. It remains to be seen if the proposed rate changes are consistent with other constraints, such as future nuclear expriments, new grains similar to $\mathrm{OC} 2$, or the production of ${ }^{17} \mathrm{O}$ in other stellar types.

We note that using Salpeter's initial mass function, stars with mass from 5 to $7 M_{\odot}$ represent $\simeq 5 \%$ of all stars. Thus, one might expect such a proportion of presolar grains from IM- relatively to 
LM-AGB stars. In principle, any grain with ${ }^{18} \mathrm{O} /{ }^{16} \mathrm{O}<10^{-4}$ and ${ }^{26} \mathrm{Mg}$ excesses is a candidate for an IM-AGB origin. There are 69 oxide grains in our database found by measurements of both ${ }^{17} \mathrm{O} /{ }^{16} \mathrm{O}$ and ${ }^{18} \mathrm{O} /{ }^{16} \mathrm{O}$ (thus representing an unbiased sample) and showing ${ }^{26} \mathrm{Mg}$ excesses. Of these, three have ${ }^{18} \mathrm{O} /{ }^{16} \mathrm{O}$ lower than $10^{-4}$. Thus, at most $\simeq 4 \%$ of presolar oxides appear to have even the possibility to have formed in IM-AGB stars. Bernatowicz et al. (2005) discuss detailed calculations of graphite and TiC grain growth in carbon stars. Their Fig. 8 indicates that larger grains form in lower mass stars. Nuth et al. (2006) propose a mechanism for the growth of crystals in AGB stars and red giants and show that only in low-mass $\left(<3 M_{\odot}\right)$ stars it is possible to form the large crystals found in primitive meteorites. Most of the oxide grains with both $\mathrm{O}$ and $\mathrm{Mg}$ measurements are larger than 1 micron, thus it is quite possible that our dataset is biased towards grains from low mass stars.

Further analysis of presolar spinel grains may identify additional grains with isotopic compositions similar to OC2 and give more precise measurements of their $\mathrm{Fe}$ and $\mathrm{Cr}$ isotopic compositions. This will provide the opportunity to test the findings of the present work as well as the possibility to constrain massive AGB models using presolar grains.

Acknowledgements. M.L. gratefully acknowledges the support of NWO through the VENI grant and thanks the members of the stellar group of Onno Pols at Utrecht University for discussion. All computations were performed on Canadian Institute for Theoretical Astrophysics's Mckenzie cluster which was funded by the Canada Foundation for Innovation and the Ontario Innovation Trust. The work of LRN and CA was supported by NASA. This work was partially supported by the Australian Research Council. We thank the anonymous referee for a thorough report, which helped to improve the paper. Finally, we thank Maurizio Busso for the original suggestion that OC2 shows the signature of an IM-AGB origin.

\section{References}

Alexander, C. M. O’D. 1997, in Astrophysical Implications of the Laboratory Study of Presolar Materials, ed. T. J. Bernatowicz, \& E. Zinner (Woodbury), AIP Conf. Proc. 402, 567

Anders, E., \& Grevesse, N. 1989, Geochim. Cosmochim. Acta, 53, 197

Angulo, C., Arnould, M., Rayet, M., et al. 1999, Nucl. Phys. A, 656, 3 (NACRE)

Asplund, M., Grevesse, N., \& Sauval, A. J. 2005, in Cosmic Abundances as Records of Stellar Evolution and Nucleosynthesis in honor of David L. Lambert, ed. T. G. Barnes III, \& F. N. Bash (San Francisco: Astronomical Society of the Pacific) 336,25

Arnould, M., Meynet, G., \& Paulus, G. 1997, in Astrophysical Implications of the Laboratory Study of Presolar Materials, ed. T. J. Bernatowicz, \& E. Zinner, (Woodbury), AIP Conf. Proc. 402, 179

Bernatowicz, T. J., Akande, O. W., Croat, T. K., \& Cowsik, R. 2005, ApJ, 631, 988

Blackmon, J. C., Champagne, A. E., Hofstee, M. A., et al. 1995, Phys. Rev. Lett., 74,2642

Boothroyd, A. I., Sackmann, I.-J., \& Ahern, S. C. 1993, ApJ, 416, 762

Cannon, R. C. 1993, MNRAS, 263, 817

Chafa, A., Tatischeff, V., Aguer, P., et al. 2005, Phys. Rev. Lett., 95, 1101

Choi, B.-G., Huss, G. R., \& Wasserburg, G. J. 1998, Science, 282, 1282

Choi, B., Wasserburg, G. J., \& Huss, G. R. 1999, ApJ, 522, L133

Clayton, D. D., \& Nittler, L. R. 2004, ARA\&A, 42, 39

Denissenkov, P. A., \& VandenBerg, D. A., 2003, ApJ, 593, 509

Fox, C., et al., 2005, Phys. Rev. C, 71, 055801

Frost, C. A., \& Lattanzio, J. C. 1996, ApJ, 473, 383

Frost, C. A., Cannon, R. C., Lattanzio, J. C., Wood, P. R., \& Forestini, M. 1998, A\&A, 332, L17

Gallino, R., Arlandini, C., Busso, M., et al. 1998, ApJ, 497, 388

García-Hernández, D. A., García-Lario, P., Plez, B., et al. 2006, A\&A, accepted [arXiv:astro-ph/0609106]

Heger, A., Langer, N., \& Woosley, S. E. 2000, ApJ, 528, 368

Herwig, F. 2004a, ApJS, 155, 651

Herwig, F. 2004b, ApJ, 605, 425

Herwig, F. 2005, ARA\&A, 43, 435

Herwig, F., \& Austin, S. M. 2004, ApJ, 613, L73

Herwig, F., Austin, S. M., \& Lattanzio, J. C. 2006, Phys. Rev. C, 73, 025802
Hillion F., Daigne B., Girard F., Slodzian G., \& Schuhmacher M. 1994, in Secondary Ion Mass Spectrometry, Proceedings SIMS IX, eds. A. Benninghoven, Y. Nihei, R. Shimizu, \& H. W. Werner (Chichester: John Wiley \& Sons), 254

Izzard, R. G., Lugaro, M., Karakas, A. I., \& Iliadis, C. 2006, in Nuclei in the Cosmos IX, Proc. of Science, PoS(NIC-IX)038

Jones, A. P., Tielens, A. G. G. M., Hollenbach, D. J., \& McKee, C. F. 1997, in Astrophysical implications of the laboratory study of presolar materials, eds. T. J. Bernatowicz, \& E. Zinner, (Woodbury), AIP Conf. Proc., 402, 595

José, J., Hernanz, M., Amari, S., Lodders, K., \& Zinner, E. 2004, ApJ, 612, 414 Karakas, A. I., \& Lattanzio, J. C. 2003, PASA, 20, 279

Karakas, A. I., Lattanzio, J. C., \& Pols, O. R. 2002, PASA, 19, 515

Karakas, A. I., Lugaro, M., \& Gallino, R. 2006a, ApJL, submitted

Karakas, A. I., Lugaro, M., Wiescher, M., Goerres, J., \& Ugalde, C. 2006b, ApJ, 643,471

Landré, V., Prantzos, N., Aguer, P., et al. 1990, A\&A, 240, 85

Limongi, M., \& Chieffi, A. 2003, ApJ, 592, 404

Lodders, K. 2003, ApJ, 591, 1220

Lugaro, M. 2005, Stardust from Meteorites: An Introduction to Presolar Grains, Series in Astronomy and Astrophysics, 9 (Singapore: World Scientific)

Lugaro, M., Davis, A. M., Gallino, R., Savina, M. R., \& Pellin, M. J. 2004a, MemSAIt, 75, 723

Lugaro, M., Ugalde, C., Karakas, A. I., et al. 2004b, ApJ, 615, 934

LUNA collaboration, Phys. Lett. B, 634, 483

Maeder, A., \& Meynet, G. 2005, A\&A, 440, 1041

Mazzitelli, I., D’Antona, F., \& Ventura, P. 1999, A\&A, 348, 846

Messenger, S., Keller, L. P., Stadermann, F. J., Walker, R. M., \& Zinner, E. 2003, Science, 300, 105

Mostefaoui, S., \& Hoppe, P. 2004, ApJ, 613, L149

Nguyen A. N., \& Zinner E. 2004, Science, 303, 1496

Nguyen, A. Zinner, E., \& Lewis, R. S. 2003, PASA, 20, 382

Nittler, L. R. 1997, in Astrophysical implications of the laboratory study of presolar materials, eds. T. J. Bernatowicz, \& E. Zinner, (Woodbury) AIP Conf. Proc. 402, 59

Nittler, L. R., \& Alexander, C. M. O’D. 1999, Lunar Planet. Sci., 30, 2041

Nittler, L. R., Alexander, C. M. O’D., Gao, X., Walker, R. M., \& Zinner, E. K. 1994, Nature, 370, 443

Nittler, L. R., Alexander, C. M. O’D., Gao, X., Walker, R. M., \& Zinner, E. 1997, ApJ, 483, 475

Nittler, L. R., Alexander, C. M. O’D., Stadermann, F. J., \& Zinner, E. 2005, Lunar Planet. Sci., 36, 2200

Nollett, K. M., Busso, M., \& Wasserburg, G. J. 2003, ApJ, 582, 1036

Nuth, J. A., III, Wilkinson, G. M., Johnson, N. M., \& Dwyer, M. 2006, ApJ, 644, 1164

Plez, B., Smith, V. V., \& Lambert, D. L. 1993, ApJ, 418, 812

Prantzos, N., Aubert, O., \& Audouze, J. 1996, A\&A, 309, 760

Rauscher, T., Heger, A., Hoffman, R. D., \& Woosley, S. E. 2002, ApJ, 576, 323

Reddy, B. E., Tomkin, J., Lambert, D. L., \& Allende Prieto, C. 2003, MNRAS, 340,304

Romano, D., \& Matteucci, F. 2003, MNRAS, 342, 185

Runkle, R. C., Champagne, A. E., Angulo, C., et al. 2005, Phys. Rev. Lett., 94 082503

Smith, V. V., Plez, B., Lambert, D. L., \& Lubowich, D. A. 1995, ApJ, 441, 735

Talon, S., \& Charbonnel, C. 2005, A\&A, 440, 981

Thielemann, F.-K., Arnould, M., \& Truran, J. W. 1986, in Advances in Nuclear Astrophysics, eds. E. Vangioni-Flam, et al. (Gif-sur-Yvette: Éditions Frontières), 525

Timmes, F. X., Woosley, S. E., \& Weaver, T. A. 1995, ApJS, 98, 617

van Loon, J. Th., Zijlstra, A. A., \& Groenewegen, M. A. T. 1999, A\&A, 346, 805

van Loon, J. Th., Cioni, M.-R. L., Zijlstra, A. A., \& Loup, C. 2005, A\&A, 438, 273

Vassiliadis, C., \& Wood, P. R. 1993, ApJ, 413, 641

Ventura, P., \& D'Antona, F. 2005a, A\&A, 439, 1075

Ventura, P., \& D'Antona, F. 2005b, A\&A, 431, 279

Weiss, A., Serenelli, A., Kitsikis, A., Schlattl, H., \& Christensen-Dalsgaard, J. 2005, A\&A, 441, 1129

Whittet, D. C. B. 1992, Dust in the galactic environment (New York: Institute of Physics)

Williams, P. M., van der Hucht, K. A., \& The, P. S. 1987, A\&A, 182, 91

Wood, P. R., Bessell, M. S., \& Fox, M. W. 1983, ApJ, 272, 99

Yoon, S.-C. \& Langer, N. 2005, A\&A, 443, 643

Zinner, E. 1998, Annual Review of Earth and Planetary Sciences, 26, 147

Zinner, E., Amari, S., Guinness, R., et al. 2003, Geochim. Cosmochim. Acta, 67, 5083

Zinner, E., Nittler, L. R., Hoppe, P., et al. 2005, Geochim. Cosmochim. Acta, 69, 4149 